\title{
Heterotopic pregnancy. Presentation of four cases
}

\author{
Enrique Herrera, MD², Eduardo Otero, MD², Luis Carlos Hincapié, MD², \\ Rafael Camacho, MD², Gustavo Gómez, MD², Carlos Humberto Quintero, MD², \\ Célico Guzmán, MD ${ }^{3}$, Luis Fernando Paz, MD, Paola Otero, MD ${ }^{4}$
}

\section{SUMMARY}

Heterotopic pregnancy is defined as intrauterine and extrauterine pregnancy entity coexisting simultaneously and which has been on the rise in recent years with the development of assisted reproductive techniques. We report 4 cases of heterotopic pregnancy, three of them resulting from assisted reproductive technologies and a spontaneous case. We also describe the methods used for diagnosis, therapies and behavior and perinatal outcome.

Keywords: Heterotopic pregnancy; Atypical ectopic; Assisted reproductive complications.

Colomb Med. 2011; 42: 518-22

\section{Embarazo heterotópico. Presentación de cuatro casos}

\section{RESUMEN}

El embarazo heterotópico se define como la gestación intrauterina y extrauterina que coexisten de forma simultánea, entidad que ha venido en aumento en los últimos años con el desarrollo de las técnicas de reproducción asistida. Se presenta 4 casos de embarazo heterotópico, tres de ellos resultado de técnicas de reproducción asistida y otro espontáneo; se describen los métodos usados para el diagnóstico, conductas terapéuticas, así como el resultado perinatal.

Palabras clave: Embarazo heterotópico; Ectópico atípico; Complicaciones reproducción asistida.

Colomb Med. 2011; 42: 518-22

Heterotopic pregnancy is the combination of intra and extra-uterine pregnancy, the first case was reported by Duverney in 1708 during an autopsy. The reported incidence in 1948 was 1:30,000 pregnancies. There are recent references with numbers ranging from 1:1300 to 1:100 pregnancies. This increased frequency is associated with increased use of assisted reproduction techniques in the medical practice. Indeed, the incidence of combined pregnancies can be up to $1 \%$ when using this technology ${ }^{1,2}$. Another important factor is the increase of pelvic inflammatory disease, which is clearly associated with extrauterine pregnancy ${ }^{3}$. In patients without risk factors, it remains an exotic obstetric event.

Approximately $80 \%$ of heterotopic pregnancies end during the first trimester, so advanced pregnancies are extremely rare. Literature reports the survival of a heterotopic pregnancy carried to term with good

1. Professor, Department of Obstetric and Gynecology, Faculty of Health, Universidad del Valle, Cali, Colombia. e-mail: enriqueherrerac@hotmail.com

2. Gynecologist, Reproductive Medicine Unit, Centro Médico Imbanaco, Cali, Colombia. e-mail: eotero@imbanaco.com.co luis.hincapie@imbanaco.com.corramacho1@yahoo.com gusgomez@cooreounivalle.edu. cochquintero@imbanaco.com.co

3. Third-Year Resident, Department of Obstetrics and Gynecology, Universidad Sur Colombiana, Neiva, Colombia. e-mail: cguzman14@hotmail.com

4. Third-Year Resident, Department of Obstetrics and Gynecology, Faculty of Health, Universidad del Valle, Cali, Colombia. e-mail: ferchopaz1409@hotmail.com p_andreaotero@hotmail.com

Received for publication January 20, 2010 Accepted for publication October 19, 2010 
perinatal outcome for both the intrauterine and extrauterine fetuses being an exceptional case ${ }^{4}$. In this work, we present four cases; three of them related to assisted reproductive technologies and other spontaneous pregnancies, requiring individualized management and achieving viable products from intrauterine pregnancies with acceptable perinatal outcomes.

\section{CASE 1}

Mixed race, 34 year-old patient, without prior major gynecological history, attended at the service of Reproductive Medicine because of primary infertility. Commitment in the male factor was found given by azoospermia, microsurgical sperm aspiration (MESA) plus intracytoplasmic sperm injection (ICSI) are considered. Andrology performed MESA and initiated a protocol of ovulation induction with follicle stimulating hormone (FSH) $200 \mathrm{U}$ and human menopausal gonadotropin (HMG) $150 \mathrm{U}$, acquiring adequate follicular growth, 36 hours prior to suction 10,000U of human chorionic gonadotropin (HCG) were administered, aspirating 5 eggs.

ICSI was performed with sperm collected from epididymis. Five embryos were transferred, achieving pregnancy. Ultrasound control was performed at 9 weeks, finding dichorionic-diamniotic twin pregnancy with decidual hematoma of the first sac and multiple echo mixed images compatible with blood clots in the endocervical canal.

At week 15, a new ultrasound control was made, finding heterotopic triplet pregnancy, two intrauterine and one ectopic cervical (Figures 1, 2), sac aspiration was performed and subsequent injection of $10 \mathrm{ml}$ of potassium chloride in ectopic cervical. Patient presented vaginal bleeding, requiring cauterization without compromise of the intrauterine fetuses. Two-week rest was recommended, ultrasound control at three weeks evidenced reduction of intracervical sac size, disappearance of clots, and proper growth of intrauterine twin pregnancy.

Ultrasound monitoring evidenced adequate fetal growth. By week 27 of gestation, the patient developed severe pre-eclampsia, reason why emergency Caesarean section was performed obtaining newborn \#1, male at $1000 \mathrm{~g}$, and \#2, female at $705 \mathrm{~g}$, which were transferred to the neonatal intensive care unit(ICU) with appropriate evolution. As Intraoperative complication, the patient presented uterine atony and placenta accreta confirmed by histology, which required hysterectomy and postoperative ICU for five days with favorable results.

\section{CASE 2}

Mixed race, 39 year-old patient, without prior medical or gynecological history of importance, attended at the service of Reproductive Medicine of Centro Médico Imbanaco in Cali, because of primary infertility. Tubo-peritoneal factor was found, compromised by left hydrosalpinx, in vitro fertilization(IVF) was considered. Ovulation induction protocol was started with FSH 200 U and HMG $75 \mathrm{U}$, achieving adequate follicular growth; 36 hours before follicular aspiration, 10,000 U of HCG were administered, aspirating three oocytes. IVF was made, obtaining three embryos, which were transferred 48 hours post- insemination, achieving pregnancy. At eight weeks of gestation, transvaginal ultrasound was made, evidencing ectopic pregnancy at the level of the right adnexa and unique intrauterine. Laparotomy surgery was performed, finding right ampullary isthmic ectopic pregnancy, $100 \mathrm{ml}$ hemoperitoneum. Right partial salpingectomy and drainage of hemoperitoneum was carried out; post-surgical evolution was satisfactory.

Ultrasound follow-up evidenced proper fetal growth. Normal common peroneal nerve (CPN) was noted until full term. Because of dystocia, the patient underwent elective cesarean surgery obtaining a newborn male infant, weighing 2890 g. Adequate post-surgical evolution was observed.

\section{CASE 3}

Mixed race, 29 year-old patient, with history of left endometrioma managed with conservative surgery; the patient consulted the Reproductive Medicine Unit at Centro Médico Imbanaco for primary infertility, associated with male factor commitment; performance of IVF + ICSI was considered, ovulation protocol was initiated with FSH achieving follicular development and obtaining three oocytes. IVF + ICSI were performed and three embryos were transferred. At week seven of pregnancy, ultrasound was made finding left adnexal heterotopic pregnancy. Open surgery was performed, 


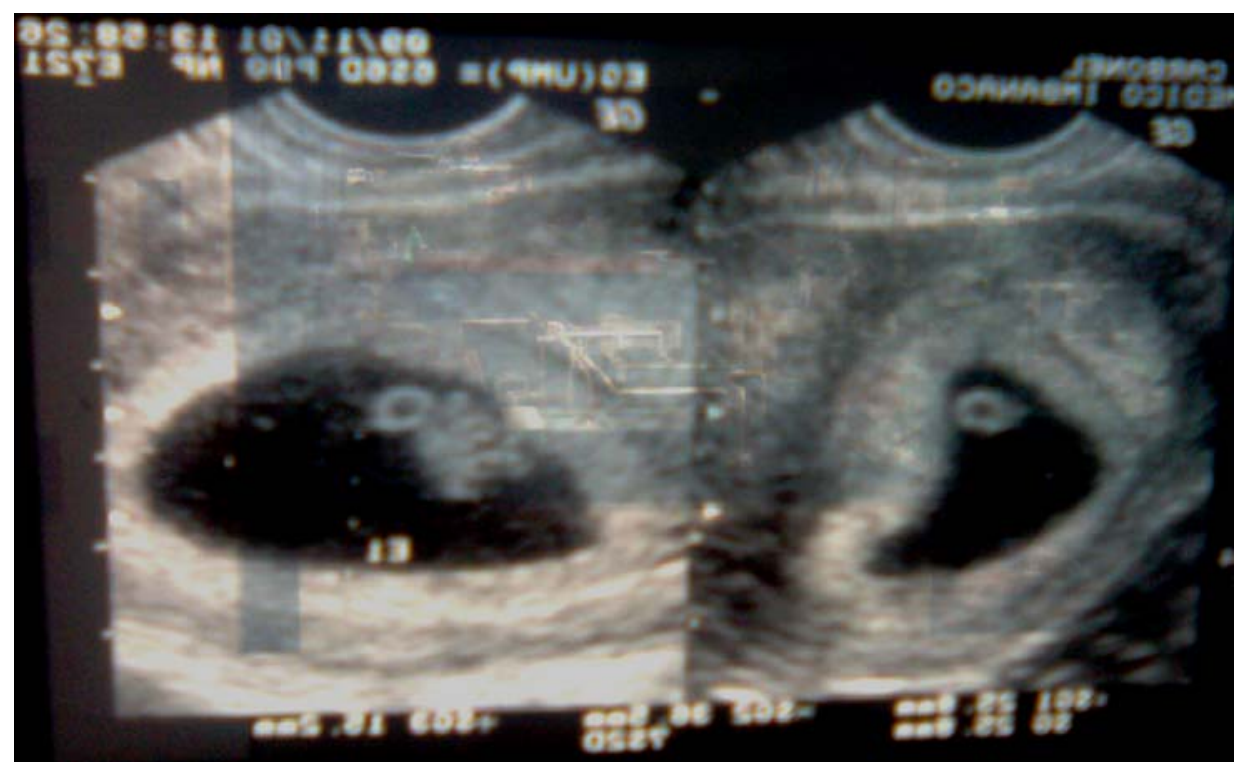

Figure 1. Ultrasound of cervical heterotopic twin pregnancy

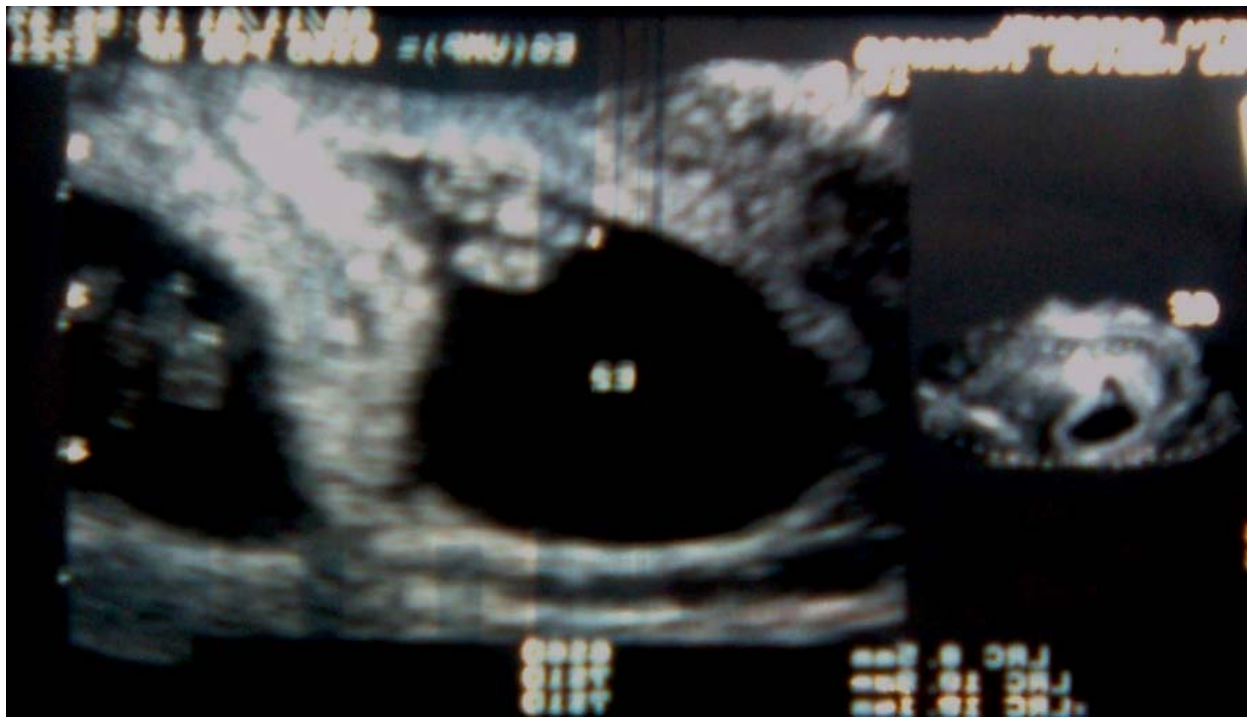

Figure 2. Ultrasound of cervical pregnancy

finding left tubal pregnancy at isthmic-cornual level, left salpingectomy was carried out with adequate postsurgical evolution, continuing intrauterine pregnancy until 37-week gestation, terminated because of intrauterine growth retardation(IUGR); cesarean section was performed obtaining a female infant, weighing 2280 g without complications.

\section{CASE 4}

Mixed race, 33 year-old patient, without prior major medical or gynecological history; she presented stabbing abdominal pain in mesogastrium radiating to the rest of the abdomen, associated with emesis, hyporexia, general malaise, chills and dizziness with menstrual failure of seven weeks two days. Physical examination revealed: Acute abdomen and vaginal tact with pelvic neck pain, normal size uterus, sensation of mass in right adnexa, qualitative HCG positive and transvaginal pelvic ultrasound showed intrauterine pregnancy with gestational age of eight weeks six days, also enlarged right adnexa with increased vascular flow, not ruling out ectopic 


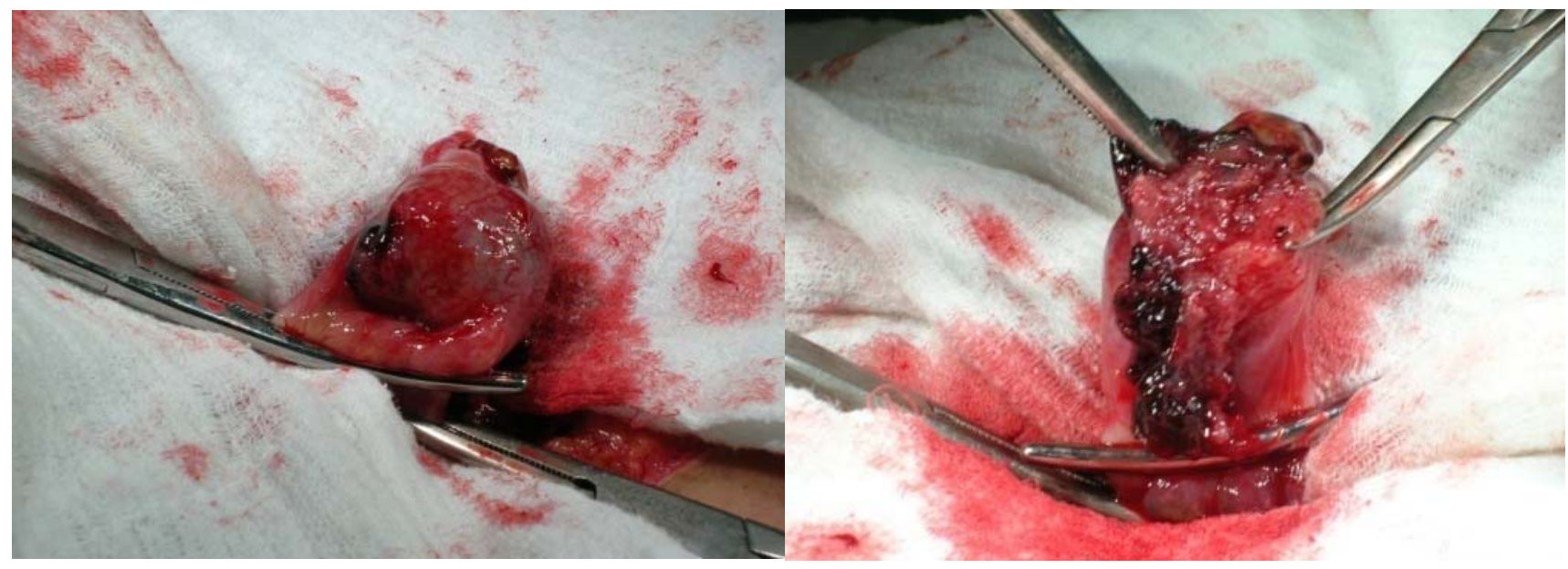

Figure 3. Surgical specimen of spontaneous heterotopic pregnancy taken to laparotomy

pregnancy, free fluid in cul-de-sac. With a diagnosis of heterotopic pregnancy (Figure 3), laparotomy was carried out, finding ruptured ampullar right ectopic pregnancy, gravid uterus augmented in size for eight weeks, right salpingectomy was performed without complications; ultrasound monitoring indicated proper fetal growth. The patient had normal prenatal control, followed by vaginal delivery at full term obtaining a female newborn of $2860 \mathrm{~g}$, without complications.

\section{DISCUSSION}

The incidence of heterotopic pregnancy has increased over the last 50 years, being 400 times more common when using assisted reproductive techniques compared with spontaneous pregnancies. It has also been observed how the transfer of four embryos increases the risk of heterotopic pregnancy. The overall risk secondary to in vitro fertilization with transfer of four embryos was estimated at 1:119 pregnancies, the transfer of more than four embryos adjusts this figure to 1:45 pregnancies ${ }^{5}$.

Many of the risk factors are shared with those referred for ectopic pregnancy, like pelvic inflammatory disease, potential etiologic factor in about $45 \%$ of cases of ectopic pregnancy. Intrinsic embryonic migration phenomena and post transfer mechanical phenomena may be involved and promote heterotopic locations. Other possible factors are superfecundation, superfetation, and ovarian hyperstimulation.

Usually, the diagnosis is made by imaging; transvaginal ultrasound can describe an adnexal mass, enabling the diagnosis of ectopic pregnancy when the gestational sac and fetal pole are present; however, ectopic classic findings are seen only in $49 \%$ of the cases. Currently, laparoscopy is gaining ground as a diagnostic and therapeutic method because of the complexity and sometimes difficult diagnosis ${ }^{6}$. It should be noted that high $\mathrm{CO}_{2}$ concentrations used may have deleterious effects on intrauterine embryo and is also a potential risk for the mother with lesions to surrounding organs or the inherited risks of pneumoperitoneum ${ }^{7,8}$, the most common images visualized by ultrasound are adnexal mass and free fluid in the cul-de-sac, in these cases it may be difficult to distinguish an ectopic pregnancy from a corpus luteum cyst, an endometrioma, or abscess.

Clinically, heterotopic pregnancy should be suspected in the presence of:

- Pregnancy with prior history of assisted reproduction techniques.

- Persistence of symptoms and signs of pregnancy after obstetric curettage.

- Persistence of high rates of HCG after an abortion.

- Clinical symptoms of a surgical abdomen with adnexal mass and intrauterine pregnancy.

In these cases, the procedure is surgical and the possibilities depend on gestational age and the site of implantation; medical treatment is banned due to the viability of intrauterine pregnancy ${ }^{4}$. Successful surgical resection of ectopic pregnancy has been described by referring lost intrauterine pregnancy up to $40 \%$. In this series of cases, different management that included different techniques like traditional salpingectomy and 
other conservative techniques like the potassium chloride injection in cervical pregnancy ${ }^{10}$, a technique successfully used by other authors to avoid radical procedures such as hysterectomy ${ }^{5-9}$.

We conclude that heterotopic pregnancy with no risk factors continues to be rare, but the diagnosis should always be taken into account, given the good perinatal outcome. Ultrasound is the diagnostic method of choice in this entity. Treatment is always surgical and can be radical or conservative, making it difficult to choose the surgical route (laparoscopic vs. open) due to factors including surgical ability, availability, and technical problems of open surgery may be preferable in cases of intra-abdominal locations and adnexal, although there are reports of successful management by laparoscopic surgery ${ }^{6}$. Laparoscopic surgery could expose the intrauterine fetus to high concentrations of $\mathrm{CO}_{2}$ and reactive oxidative substances with higher probability of embryoitoxicity ${ }^{8}$. When heterotopic pregnancy is implanted in the posterior cul-de-sac and cervix, extraction of ectopic and spontaneous evolution of intrauterine is feasible, which is always exposed to complications among which is the stillbirth.

Conflict of interest. None of the authors has conflicts of interest related to this study.

\section{ACKNOWLEDGEMENTS}

We thank the staff of the Reproductive Medicine Unit at the Centro Médico Imbanaco for their assistance in gathering information, as well as the Department of Gynecology and Obstetrics at Universidad del Valle for its assistance in the presentation of this series.

\section{REFERENCES}

1. Condous G. Ectopic pregnancy risk factors and diagnosis. Aust Fam Physician. 2006; 35: 854-7.

2. Ludwig M, Kaisi M, Bauer O. Heterotopic pregnancy in a spontaneous cycle: do not forget about it! Eur J Obstet Gynecol Reprod Biol. 1999; 87: 91-3.

3. Kamwendo F, Forslin L, Bodin L, Danielsson D. Epidemiology of ectopic pregnancy during a 28 year period and the role of pelvic inflammatory disease. Sex Transm Inf. 2000; 76: 28-32.

4. Ludwig M, Kaisi M, Bauer O, Diedrich K. The forgotten child: a case of heterotopic, intraabominal and intrauterine pregnancy carried to term. Hum Reprod. 1999; 14: 1372-4.

5. Massoc P, Quiroz V, Díaz E. Embarazo triple heterotópico. Rev Chil Obstet Ginecol. 2000; 65: 212-4.

6. Louis-Sylvestre C, Morice P, Chapron C, Dubuisson J. The role of laparoscopy in the diagnosis and management of heterotopic pregnancies. Hum Reprod. 1997; 12: 1100-2.

7. Steinbrook RA. Anaesthesia, minimally invasive surgery and pregnancy. Best Pract Res Clin Anaesthesiol. 2002; 16: 13143.

8. Nezhat C, Seidman DS, Vreman HJ, Stevenson DK, BrockUtne JG, Nezhat F. The risk of carbon monoxide poisoning after prolonged laparoscopic surgery. Obstet Gynecol. 1996; 88: 771-4.

9. Guntiñas A, Charines E, De Andrés P, Herrero F, Rodríguez R, González A. Heteroptopic cervical pregnancy: A case report. Progr Obstetr Ginecol. 2001; 44: 346-9.

10. Milenko Prorocic Treatment of heterotopic cervical pregnancy after in vitro fertilization-embryo transfer by using transvaginal ecography-guided aspiration and instillation of hypertonic solution of sodium chloride. Fertil Steril. 2007; 88: 969-75. 